\title{
Flexible Stock Allocation and Trim Loss Control for Cutting Problem in the Industrial-Use Paper Production
}

\author{
Fu-Kwun Wang and Feng-Tai Liu \\ Department of Industrial Management, National Taiwan University of Science and Technology, Taipei 106, Taiwan \\ Correspondence should be addressed to Fu-Kwun Wang; fukwun@mail.ntust.edu.tw
}

Received 29 November 2013; Accepted 25 May 2014; Published 17 June 2014

Academic Editor: Hsiao-Fan Wang

Copyright ( 2014 F.-K. Wang and F.-T. Liu. This is an open access article distributed under the Creative Commons Attribution License, which permits unrestricted use, distribution, and reproduction in any medium, provided the original work is properly cited.

\begin{abstract}
We consider a one-dimensional cutting stock problem (CSP) in which the stock widths are not used to fulfill the order but kept for use in the future for the industrial-use paper production. We present a new model based on the flexible stock allocation and trim loss control to determine the production quantity. We evaluate our approach using a real data and show that we are able to solve industrial-size problems, while also addressing common cutting considerations such as aggregation of orders, multiple stock widths, and cutting different patterns on the same machine. In addition, we compare our model with others, including trim loss minimization problem (TLMP) and cutting stock problem (CSP). The results show that the proposed model outperforms the other two models regarding total flexibility and trim loss ratio.
\end{abstract}

\section{Introduction}

A one-dimensional cutting stock problem (CSP) is one of the famous combinatorial optimization problems, which has many applications in industries, such as paper, wood, textiles, steel, space, ship construction, and logistic transportation [1-6]. Most studies focus on minimizing the trim loss that is the amount of residual pieces of processed stock lengths. A standard one-dimensional cutting stock problem (S1DCSP) as a kind of the above problems is known as an NPcomplete one [7]. Numerous studies have examined how to fulfill orders and optimize production planning. Gilmore and Gomory [8] presented a delayed pattern generation technique for solving a one-dimensional cutting problem using linear programming. Other methods, including pattern-oriented approach, item-oriented approach, mixed approach and exact approach, can be found in [9-27].

In the industrial-use paper industry, the production quantity is usually greater than the customers' order. Using the traditional CSP, the trim loss can be significant. Thus, we need to consider usable leftovers to prevent the trim loss generated after optimization. This issue becomes a onedimensional CSP with usable leftovers. Yanasse [28] reported that the literature on usable leftovers is scarce and the problem still lacks clear and appropriate definitions. Kos and Duhovnik [29] proposed usable leftover material used in the next cutting plan to reduce trim loss. Related studies can be found in [6, 29-35]. Cherri et al. [31] presented several modifications in some well-known heuristics to solve a onedimensional CSP with usable leftovers. Poldi and Arenales [32] presented a study with the classical one-dimensional integer CSP, which consists of cutting a set of available stock lengths in order to produce smaller ordered items. Cui and Yang [33] considered a one-dimensional CSP with useful leftover in the cutting plan. Cherri et al. [35] proposed a priority-in-use heuristic approach to solve a one-dimensional CSP with usable leftovers. However, these models cannot be directly used for solving the CSP in the industrial-use production that each reel can only be produced a certain number of rolls depending on its cutting machine. Wang and Liu [36] presented a new decision model for reducing trim loss and inventory in the paper industry.

In this study, we present a new model based on the flexible stock allocation and trim loss control to determine the production quantity. Our proposed model is a flexibility maximization problem (FAP). Under a certain condition of trim loss control, FAP can be confined to cutting stock problem (CSP) or trim loss minimization problem (TLMP). 
The remainder of this paper is organized as follows. In Section 2, the definition of problem in the paper industry is presented. A new model is developed in Section 3. In Section 4, some examples illustrate the application of the proposed model. Finally, conclusions are drawn in Section 5.

\section{Problem Definition}

The production of industrial-use paper starts from raw material to reels and then from reels to the production of rolls as finished goods. The entire operation mode is cyclical production, which is the only method for achieving production efficiency. Therefore, the leftover material is not used in a follow-up production. For the production planning (see Figure 1), the customer's paper requirements are obtained and the marketing demand is predicted. Then, during the combined production-marketing meeting, the number of production days and the production quantity of paper types are determined. The production quantity indicates the $N$ number of reels, and each reel can produce the NR number of rolls that depends on the paper type. It should be noted that the unit of the paper width is millimeter $(\mathrm{mm})$.

To formulate the models of CSP, TLMP and FAP, see the notations section are used.

The main research question is how to improve the stock allocation and trim loss of a CSP with useful leftovers in the paper industry. This problem can be studied for either one- or multidimensional CSPs. In this study, one-dimensional CSP with useful leftovers was used. We first provide two examples to illustrate the differences between CSP, TLMP, and manual adjustment (MA). In practice, MA is used in the industrialuse paper industry in which the CSP and TLMP solutions are candidates as manually selecting as MA solution. The CSP and TLMP are usually solved through column generation $[8,9]$. To obtain the solutions of CSP and TLMP, the computer program was written in Lingo 11 Software [37].

The formulation of CSP is defined as follows:

$$
\text { (CSP) Minimize } \sum_{r=1}^{t} x_{r}
$$

s.t.

$$
\begin{gathered}
L \geq \sum_{i=1}^{m} a_{i r} \mathrm{Ow}_{i}, \quad \text { (width of reel constraint) } \\
\sum_{r=1}^{t} a_{i r} x_{r} \geq d_{i}, \quad \text { (demand constraint) } \\
\mathrm{UB} \geq L-\sum_{i=1}^{m} a_{i r} \mathrm{ow}_{i}, \quad \text { (trim loss constraint) }
\end{gathered}
$$

where $a_{i r}$ and $x_{r}$ are decision variables and integer variables. Minimizing the total number of patterns is the objective function (1) of the model. Constraint (2) guarantees the cutting stocks regarding the reel width. Constraint (3) guarantees the cutting stocks regarding the demand. In the industrial-use paper production, there exists the maximum trim loss for each cutting, and then constraint (4) guarantees the waste of each roll during the cutting process.

In order to reduce the trim loss, a modified model called TLMP is given as follows:

$$
\text { (TLMP) Minimize } \quad \sum_{r=1}^{t}\left(L-\sum_{i=1}^{m} a_{i r} \mathrm{ow}_{i}\right) x_{r},
$$

s.t.

$$
\begin{gathered}
L \geq \sum_{i=1}^{m} a_{i r} \mathrm{Ow}_{i}, \quad \text { (width of reel constraint) } \\
\sum_{r=1}^{t} a_{i r} x_{r} \geq d_{i}, \quad \text { (demand constraint) } \\
\mathrm{SRQ}=\frac{\sum_{r=1}^{t} x_{r}}{\mathrm{NR}} \quad \text { (reel set constraint) } \\
\mathrm{UB} \geq L-\sum_{i=1}^{m} a_{i r} d_{i}, \quad \text { (trim loss constraint) }
\end{gathered}
$$

where $a_{i r}$ and $x_{r}$ are decision variables and integer variables. Minimizing the total trim loss is the objective function (5) of the model. Constraint (6) guarantees the cutting stocks regarding the reel width. Constraint (7) guarantees the cutting stocks regarding the demand. In industry paper production, the maximum trim loss for each cutting and the limit production volume are considered; then constraint (8) guarantees the number of rolls for each reel, and constraint (9) guarantees the waste of each roll during the cutting process.

For example 1, we assume that the reel width is 10 units, $\mathrm{NR}$ is $3, \mathrm{UB}$ is 3 , the demand of order widths $\{3,4\}$ is $\{3,3\}$, and the stock widths are $\{3,4,5\}$. In Figure 2, we provide CSP, TLMP, and MA solutions. The trim loss using CSP is 5 units. And the trim loss using TLMP is zero. We found that the stock width using CSP is obtained as $\{4\}$ and the stock widths using TLMP are $\{3\} * 3$. In order to obtain flexible stock widths, using MA based on CSP and TMLP solutions, the extending stock width is determined as $\{6\}$, and the stock width is obtained as $\{3\}$. Thus, MA can provide more flexible stock width $\{6\}$.

For example 2, we assume that the reel width is 10 units, $\mathrm{NR}$ is $3, \mathrm{UB}$ is 3 , the demand of order widths $\{3,5\}$ is $\{1,1\}$, and the stock widths are $\{3,4,5\}$. In Figure 3, we provide CSP, TLMP, and MA solutions. The trim loss using CSP is 2 units. And the trim loss using TLMP is zero. We found that the stock width using CSP is zero, the unused rolls are 2 and 3 , and the stock widths using TLMP are $\{3\} * 1,\{4\} * 1$, and $\{5\} * 3$. Using MA based on CSP and TMLP solutions, the extending stock widths are determined as $\{10,10\}$ or $\{7,10\}$, the stock width is obtained as zero or $\{5\}$, and the trim loss is obtained as 2 units or zero. Thus, MA can provide more flexible stock widths $\{10,10\}$ or $\{7,10\}$.

Based on the above discussions, we conclude that the MA approach can provide more flexible stock widths in a one-dimensional CSP with useful leftovers. This motivates 


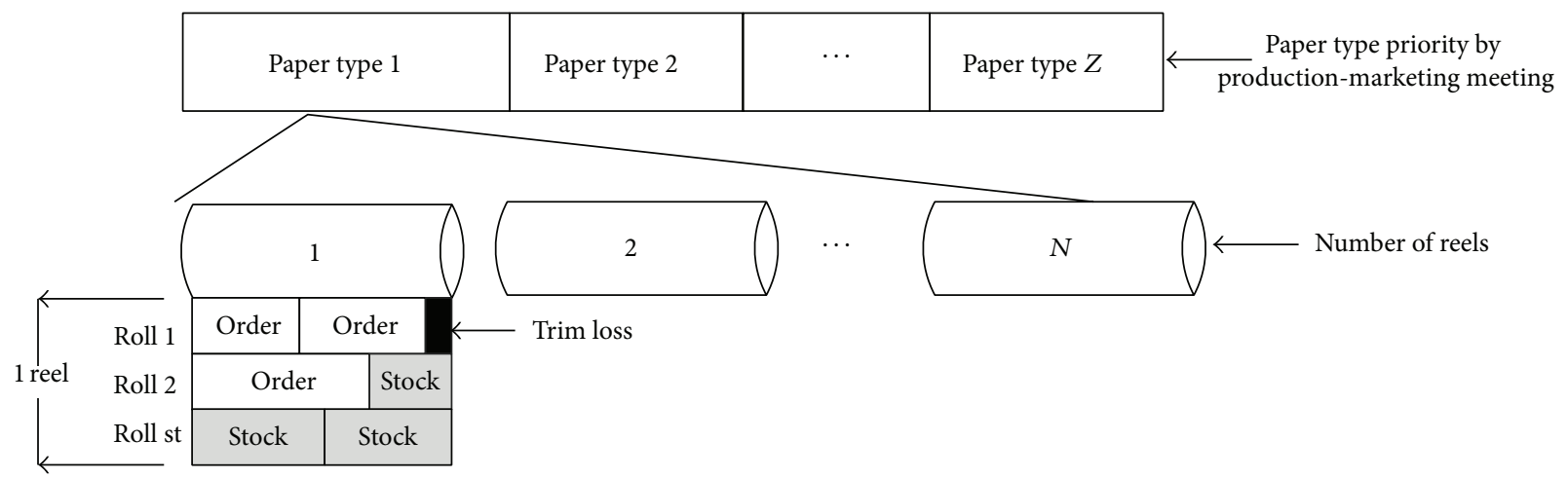

FIGURE 1: Production planning in the paper industry.

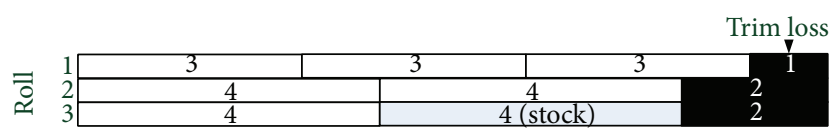

(a)

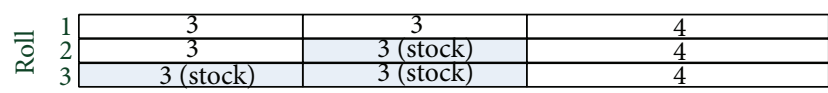

(b)

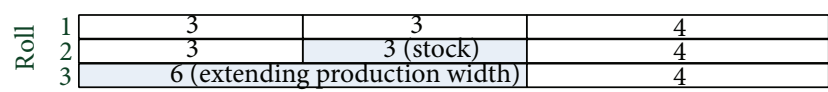

(c)

Figure 2: (a) CSP solution, (b) TLMP solution, and (c) MA solution for example 1 .

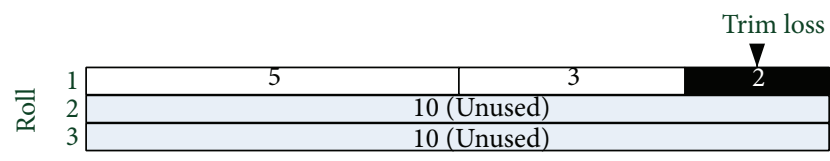

(a)

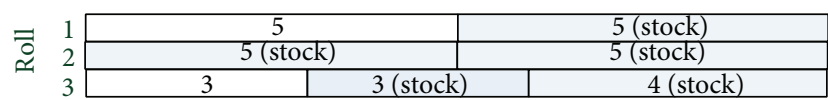

(b)

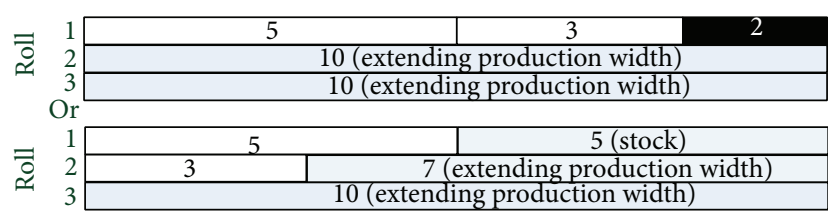

(c)

FIGURE 3: (a) CSP solution, (b) TLMP solution, and (c) MA solution for example 2 .

the development of a mathematical formulation for a onedimensional CSP with useful leftovers in the industrial-use industry.

\section{The Proposed Model}

To provide more flexible leftovers, we propose the flexible stock allocation approach. It should be noted that the concept of extending stock widths is similar to the usable leftovers [31]. In FAP, we have stock items of width $\mathrm{sw}_{i}, i=\{1, \ldots, m\}$, the reel width is $L$, each paper width is based on a fixed number called GAP to differentia, and the extending stock width can be obtained from ( $\left.\mathrm{sw}_{m}+\mathrm{GAP}\right)$ to $L$. Thus, the extending stock item of width is established as

$$
\mathrm{ew}_{e}=\left\{\mathrm{sw}_{m}+(e-m) * \mathrm{GAP}\right\},
$$

where $e=\{m+1, \ldots$, ex $\}$, ex $=m+\left(L-\mathrm{sw}_{m}\right) / \mathrm{GAP},(L-$ $\left.\mathrm{sw}_{m}\right) / \mathrm{GAP}$ is integer, and $m$ is the number of stock widths. Using (10), we can combine $\mathrm{sw}_{i}$ and $\mathrm{ew}_{e}$ as production width $\mathrm{pw}_{k}$. This new set of paper widths can provide more flexible stock allocation for cutting plan.

In order to evaluate the benefit of the flexible stock allocation, we define a flexible coefficient $f_{k}$ for each production width $\mathrm{pw}_{k}$ that is obtained as follows:

$$
f_{k}=\left\{p \mid \mathrm{pw}_{k} \geq \sum_{i=1}^{m} \mathrm{pw}_{i} y_{i z}, \mathrm{UB} \geq\left(\mathrm{pw}_{k}-\sum_{i=1}^{m} \mathrm{pw}_{i} y_{i z}\right)\right\},
$$

where $k=\{1,2, \ldots, m\}, z=\{1,2, \ldots, p\}, p$ is the number of patterns, $y_{i z}$ is the number of widths $i$ in pattern $z$, and $y_{i z}$ is a positive integer. For instance, we set $\mathrm{UB}=999$. Since $\mathrm{pw}_{1}\{1000\}$ has only one combination, the $f_{1}$ value is assigned to one. Since $\mathrm{pw}_{2}\{1100\}$ can be divided into two combinations $\{1000,1100\}$, the $f_{2}$ value is assigned to two. Furthermore, $\mathrm{pw}_{13}\{2200\}$ can be divided into fourteen combinations $\{\{1000,1000\},\{1000,1100\},\{1000,1200\},\{1100,1100\}, 1300$, $1400,1500,1600,1700,1800,1900,2000,2100,2200\}$; the $f_{13}$ value is assigned to fourteen. In addition, a flexible coefficient $f_{k}$ for each extending width is obtained as follows:

$$
f_{k}=f_{m}+(k-m),
$$

where $k=\{m+1, \ldots, e x\}$ and $e=\{m+1, \ldots, e x\}$. 
Table 1: Product information and data for $\mathrm{ow}_{i}, d_{i}$, and $\mathrm{sw}_{i}$.

\begin{tabular}{cccc}
\hline$i$ & $\mathrm{ow}_{i}$ & $d_{i}$ & $\mathrm{sw}_{i}$ \\
\hline 1 & $\mathrm{X}$ & 0 & 1000 \\
2 & $\mathrm{X}$ & 0 & 1100 \\
3 & 1200 & 7 & 1200 \\
4 & 1300 & 22 & 1300 \\
5 & $\mathrm{X}$ & 0 & 1400 \\
6 & 1500 & 28 & 1500 \\
7 & 1600 & 58 & 1600 \\
8 & 1700 & 47 & 1700 \\
9 & 1800 & 43 & 1800 \\
10 & 1900 & 7 & 1900 \\
11 & 2000 & 20 & 2000 \\
12 & 2100 & 9 & 2100 \\
13 & 2200 & 30 & 2200 \\
14 & 2300 & 7 & 2300 \\
15 & 2400 & 12 & 2400 \\
16 & 2500 & 20 & 2500 \\
\hline
\end{tabular}

Note: $\mathrm{X}=$ not available.

We introduce a coefficient $R$ for controlling the trim loss. Thus, the proposed formulation is as follows:

$$
\begin{aligned}
\operatorname{Maximize} \sum_{k=1}^{e x} & \left(\sum_{r=1}^{t} a_{k r} x_{r}-\mathrm{pq}_{k}\right) \\
& \times f_{k} \mathrm{pw}_{k}+R \sum_{r=1}^{t}\left(L-\sum_{k=1}^{e x} a_{k r} \mathrm{pw}_{k}\right) x_{r},
\end{aligned}
$$

s.t.

$$
\begin{gathered}
\sum_{k=1}^{e x} a_{k r} \mathrm{pw}_{k} \leq L \quad \text { (width of reel constraint) } \\
\mathrm{pq}_{k} \leq \sum_{r=1}^{t} a_{k r} x_{r} \quad \text { (demand constraint) } \\
\mathrm{SRQ}=\frac{\left(\sum_{r=1}^{t} x_{r}\right)}{\mathrm{NR}} \quad \text { (reel set constraint) } \\
\mathrm{L}-\sum_{k=1}^{\mathrm{ex}} a_{k r} \mathrm{pw}_{k} \leq \mathrm{UB} \quad \text { (trim loss constraint), }
\end{gathered}
$$

where $a_{k r}$ and $x_{r}$ are decision variables and integer variables. Maximizing the total flexibility is the objective function (13) of the model that is the summation of total production coefficient and total trim loss coefficient. The composition of each production coefficient includes nonorder quantity, production width, and flexible coefficient, and the trim loss coefficient includes the coefficient $R$ and the total trim loss. Constraint (14) guarantees the cutting stocks regarding the reel width. Constraint (15) guarantees the cutting stocks regarding the demand. Constraint (16) guarantees the number of rolls for each reel. Constraint (17) guarantees the waste

\begin{tabular}{|c|c|c|c|c|c|c|c|}
\hline \multirow{2}{*}{$k$} & \multirow{2}{*}{$\mathrm{pw}_{k}$} & \multirow{2}{*}{$\mathrm{pq}_{k}$} & \multirow{2}{*}{$f_{k}$} & \multicolumn{2}{|c|}{ CSP } & \multicolumn{2}{|c|}{ FAP } \\
\hline & & & & Solution $_{k}$ & Stock $_{k}$ & Solution $_{k}$ & Stock $_{k}$ \\
\hline 1 & 1000 & 0 & 1 & 31 & 31 & 21 & 21 \\
\hline 2 & 1100 & 0 & 2 & 0 & 0 & 0 & 0 \\
\hline 3 & 1200 & 7 & 3 & 7 & 0 & 12 & 5 \\
\hline 4 & 1300 & 22 & 4 & 22 & 0 & 23 & 1 \\
\hline 5 & 1400 & 0 & 5 & 0 & 0 & 10 & 10 \\
\hline 6 & 1500 & 28 & 6 & 30 & 2 & 28 & 0 \\
\hline 7 & 1600 & 58 & 7 & 58 & 0 & 58 & 0 \\
\hline 8 & 1700 & 47 & 8 & 47 & 0 & 47 & 0 \\
\hline 9 & 1800 & 43 & 9 & 43 & 0 & 43 & 0 \\
\hline 10 & 1900 & 7 & 10 & 7 & 0 & 7 & 0 \\
\hline 11 & 2000 & 20 & 11 & 20 & 0 & 20 & 0 \\
\hline 12 & 2100 & 9 & 12 & 9 & 0 & 9 & 0 \\
\hline 13 & 2200 & 30 & 14 & 30 & 0 & 30 & 0 \\
\hline 14 & 2300 & 7 & 16 & 7 & 0 & 7 & 0 \\
\hline 15 & 2400 & 12 & 19 & 12 & 0 & 12 & 0 \\
\hline 16 & 2500 & 20 & 22 & 20 & 0 & 20 & 0 \\
\hline 21 & $\# 3000$ & 0 & 27 & 0 & 0 & 1 & 1 \\
\hline 37 & $\# 4600$ & 0 & 43 & 1 & 1 & 1 & 1 \\
\hline \multicolumn{4}{|c|}{ TLR (\%) } & 3.3 & \multicolumn{3}{|c|}{1.4} \\
\hline \multicolumn{4}{|c|}{$\mathrm{TF}$} & & 86 & & 160 \\
\hline
\end{tabular}
of each roll during the cutting process.
TABLE 2: Product information and data for $\mathrm{pw}_{k}, \mathrm{pq}_{k}$, and $f_{k}$.

\begin{tabular}{cccccccc}
\hline$k$ & $\mathrm{pw}_{k}$ & $\mathrm{pq}_{k}$ & $f_{k}$ & $k$ & $\mathrm{pw}_{k}$ & $\mathrm{pq}_{k}$ & $f_{k}$ \\
\hline 1 & 1000 & 0 & 1 & 17 & $\# 2600$ & 0 & 23 \\
2 & 1100 & 0 & 2 & 18 & $\# 2700$ & 0 & 24 \\
3 & 1200 & 7 & 3 & 19 & $\# 2800$ & 0 & 25 \\
4 & 1300 & 22 & 4 & 20 & $\# 2900$ & 0 & 26 \\
5 & 1400 & 0 & 5 & 21 & $\# 3000$ & 0 & 27 \\
6 & 1500 & 28 & 6 & 22 & $\# 3100$ & 0 & 28 \\
7 & 1600 & 58 & 7 & 23 & $\# 3200$ & 0 & 29 \\
8 & 1700 & 47 & 8 & 24 & $\# 3300$ & 0 & 30 \\
9 & 1800 & 43 & 9 & 25 & $\# 3400$ & 0 & 31 \\
10 & 1900 & 7 & 10 & 26 & $\# 3500$ & 0 & 32 \\
11 & 2000 & 20 & 11 & 27 & $\# 3600$ & 0 & 33 \\
12 & 2100 & 9 & 12 & 28 & $\# 3700$ & 0 & 34 \\
13 & 2200 & 30 & 14 & 29 & $\# 3800$ & 0 & 35 \\
14 & 2300 & 7 & 16 & 30 & $\# 3900$ & 0 & 36 \\
15 & 2400 & 12 & 19 & 31 & $\# 4000$ & 0 & 37 \\
16 & 2500 & 20 & 22 & 32 & $\# 4100$ & 0 & 38 \\
& & & & 33 & $\# 4200$ & 0 & 39 \\
& & & & 34 & $\# 4300$ & 0 & 40 \\
& & & & 35 & $\# 4400$ & 0 & 41 \\
& & & & 36 & $\# 4500$ & 0 & 42 \\
& & & & 37 & $\# 4600$ & 0 & 43 \\
\hline
\end{tabular}

Note: \# = extending stock width.

TABLE 3: Optimal solutions using FAP and CSP methods.

Note: rolls $=135, R=0$, and stock $k=$ solution $_{k}-\mathrm{pq}_{k}$.

When $R=0$, the objective function (13) becomes to be a maximize function of $\sum_{k=1}^{e x}\left(\sum_{r=1}^{t} a_{k r} x_{r}-\mathrm{pq}_{k}\right) f_{k} \mathrm{pw}_{k}$; that is, 
TABLE 4: Optimal solutions using FAP and TLMP method.

\begin{tabular}{cccccccc}
\hline$k$ & \multirow{2}{*}{$\mathrm{pw}_{k}$} & \multirow{2}{*}{$\mathrm{pq}_{k}$} & $f_{k}$ & \multicolumn{2}{c}{ TLMP } & \multicolumn{2}{c}{ FAP } \\
\hline 1 & 1000 & 0 & 1 & 39 & 39 & 37 & 37 \\
2 & 1100 & 0 & 2 & 2 & 2 & 1 & 1 \\
3 & 1200 & 7 & 3 & 9 & 2 & 8 & 1 \\
4 & 1300 & 22 & 4 & 22 & 0 & 23 & 1 \\
5 & 1400 & 0 & 5 & 9 & 9 & 6 & 6 \\
6 & 1500 & 28 & 6 & 28 & 0 & 28 & 0 \\
7 & 1600 & 58 & 7 & 58 & 0 & 58 & 0 \\
8 & 1700 & 47 & 8 & 47 & 0 & 47 & 0 \\
9 & 1800 & 43 & 9 & 43 & 0 & 43 & 0 \\
10 & 1900 & 7 & 10 & 7 & 0 & 7 & 0 \\
11 & 2000 & 20 & 11 & 20 & 0 & 20 & 0 \\
12 & 2100 & 9 & 12 & 9 & 0 & 9 & 0 \\
13 & 2200 & 30 & 14 & 30 & 0 & 30 & 0 \\
14 & 2300 & 7 & 16 & 7 & 0 & 7 & 0 \\
15 & 2400 & 12 & 19 & 12 & 0 & 15 & 3 \\
16 & 2500 & 20 & 22 & 20 & 0 & 20 & 0 \\
\hline \multicolumn{6}{c}{ TLR (\%) } & \multicolumn{7}{c}{ TF } & & & & \\
\hline
\end{tabular}

Note: rolls $=135, R=-1000$, and stock $k=$ solution $_{k}-\mathrm{pq}_{k}$.

it does not consider trim loss. If the production capacity fails to satisfy (15) during the problem-solving process, a full roll is generated. Subsequently, because the flexibility of $f_{e} \mathrm{pw}_{e x}$ is greater than any of the leniency and flexibility coefficient combinations, the full roll is substituted by $\mathrm{pw}_{e x}$. In addition, the optimal CSP solution also generates a full roll and the full roll is substituted by $\mathrm{pw}_{e x}$; thus, the FAP results approximate the CSP target function; that is, Minimize $\sum_{r=1}^{t} x_{r}$. Therefore, the difference between $\operatorname{FAP}(R=0)$ and the $a_{k r}$ of CSP can be compared.

When $R=-\infty, \sum_{k=1}^{e x}\left(\sum_{r=1}^{t} a_{k r} x_{r}-\mathrm{pq}_{k}\right) f_{k} \mathrm{pw}_{k}$ can be neglected and the objective function (13) approaches Maximize $R \sum_{r=1}^{t}\left(L-\sum_{k=1}^{e x} a_{k r} \mathrm{pw}_{k}\right) x_{r}$. In addition, $R$ approximates the TLMP target function; that is, Minimize $\sum_{r=1}^{t}(L-$ $\left.\sum_{k=1}^{e x} a_{k r} \mathrm{pw}_{k}\right) x_{r}$. Therefore, the difference between $\mathrm{FAP}(R=$ $-\infty)$ and the $a_{k r}$ of TLMP can be compared.

In summary, when $R=0$, flexible stock becomes the optimal condition and trim loss is maximized. Conversely, when $R=-\infty$, flexible stock becomes the least favorable condition and trim loss is minimized. Therefore, the control of variable $R$ is a flexible stock and trim loss strategy that decision makers adopt during the production process.

\section{Illustrative Examples}

We consider a real case from an industrial-use paper production and five simulated datasets to illustrate the application of our proposed method. We set the current scheduling quantity as SRQ reels, and each reel can produce NR number of rolls. The cutting machine width limit is $L$, and the maximum trim loss is UB. These parameters are defined as NR $=3, L=$ $4600 \mathrm{~mm}, \mathrm{SRQ}=45$ reels $=135$ rolls, and UB $=999 \mathrm{~mm}$.

To obtain the solutions of CSP, TLMP, and FAP, the computer program is divided into the engine and the user interface. The engine interface was written in Lingo 11 Software [37]. The user interface in Visual Basic 5 enables the navigation of data flow from various input sources, such as a common company database and a random number dataset.

4.1. A Real Case from an Industrial-Use Paper Production. According to the FAP model in Section 3, the details are as follows.

Step 1. Define $\mathrm{ow}_{i}, d_{i}$, and $\mathrm{sw}_{i}$, for $i=1,2, \ldots, 16$ (see Table 1 ).

Step 2. Using (10) to obtain the extending widths, since GAP $=100$ and $m=16$, we can obtain that $e=$ $\{17,18, \ldots, 37\}$ and $\mathrm{ew}_{e}=\{2600,2700, \ldots, 4600\}$.

Step 3. Aggregate $d_{i}$ to $\mathrm{pq}_{k}, \mathrm{sw}_{i}$ to $\mathrm{pw}_{k}$, and $\mathrm{ew}_{e}$ to $\mathrm{pw}_{k}$, for $i=1,2, \ldots, 16$ and $k=1,2, \ldots, 37$.

Step 4. Use (11)-(12) to compute the flexible coefficient $f_{k}$ for $\mathrm{pw}_{k}$ (see Table 2).

Using FAP to perform optimization, $R$ must be set to 0 , thereby allowing FAP results to approximate those of CSP. In this case, we obtained the production capacities of FAP and CSP, stock, trim loss ratio (TLR), and total flexible coefficient (TF), where TLR $=\left[\sum_{r=1}^{t}\left(L-\sum_{k=1}^{e x} a_{k r} \mathrm{pw}_{k}\right) x_{r}\right] /\left(L \sum_{r=1}^{t} x_{r}\right) \times$ $100 \%$ and $\mathrm{TF}=\sum_{k=1}^{e x} \mathrm{pw}_{k} f_{k}$.

The primary reason for comparing CSP was to determine whether FAP effectively reduced TLR and whether the flexible stock of FAP is superior to that of CSP (see Table 3). The TLRs for CSP and FAP were 3.3 and 1.4, respectively; the flexible stocks for CSP, FAP, and extending stock were $\{\{1000,31\},\{1500,2\},\{\# 4600,1\}\},\{\{1000,21\},\{1200,5\},\{1300$, $1\},\{1400,10\},\{\# 3000,1\},\{\# 4600,1\}\}$, and $\{\{\# 3000,1\},\{\# 4600$, $1\}\}$, respectively. Thus, the results suggest that FAP outperforms CSP in reducing TLR and that the flexible stock and TF of FAP are superior to that of CSP.

To compare FAP to TLMP, the flexible variable $R$ of FAP was set to -1000 , which denotes minimal TLR. In this case, we obtained the production capacities of FAP and TLMP, stock, TLR, and TF(see Table 4).

The primary reason for comparing TLMP was to determine whether the TLR of FAP is similar to that of TLMP or not and whether the flexible stock of FAP is superior to that of TLMP or not (see Table 4). The TLRs for TLMP and FAP were 0.42 and 0.42 , respectively; the flexible stocks for TLMP and FAP were $\{\{1000,39\},\{1100,2\},\{1200,2\},\{1400,9\}\}$, and $\{\{1000,37\},\{1100,1\},\{1200,1\},\{1300,1\},\{1400,6\},\{2400,3\}\}$, respectively. Notably, the flexible stock of FAP $\{2400,3\}$ was considerably more lenient. Therefore, based on the results, the TLR of FAP was identical to that of TLMP, and the flexible stock and TF of FAP were superior to those of TLMP.

Moreover, we employed sensitivity analysis to observe the influence that $R$ has on TLR and TF. When $R=$ $1,0,-1, \ldots,-\infty$, and $R$ is an integer, the results as shown in Table 5 are obtained. 
TABLE 5: The sensitivity analysis of coefficient $R$ for stock $k_{k}$ using FAP.

\begin{tabular}{|c|c|c|c|c|c|c|c|c|c|}
\hline \multirow{2}{*}{$k$} & \multirow{2}{*}{$\mathrm{pw}_{k}$} & \multirow{2}{*}{$f_{k}$} & \multicolumn{7}{|c|}{$R$} \\
\hline & & & 1 & 0 & $-1 \sim-2$ & $-3 \sim-8$ & $-9 \sim-86$ & $-87 \sim-533$ & $-534 \sim-\infty$ \\
\hline 1 & 1000 & 1 & 11 & 21 & 22 & 28 & 36 & 37 & 37 \\
\hline 2 & 1100 & 2 & 0 & 0 & 1 & 1 & 1 & 1 & 1 \\
\hline 3 & 1200 & 3 & 5 & 5 & 4 & 1 & 1 & 1 & 1 \\
\hline 4 & 1300 & 4 & 1 & 1 & 1 & 1 & 1 & 1 & 1 \\
\hline 5 & 1400 & 5 & 10 & 10 & 10 & 10 & 6 & 6 & 6 \\
\hline 6 & 1500 & 6 & 0 & 0 & 0 & 0 & 0 & 0 & 0 \\
\hline 7 & 1600 & 7 & 0 & 0 & 0 & 0 & 0 & 0 & 0 \\
\hline 8 & 1700 & 8 & 0 & 0 & 0 & 0 & 0 & 0 & 0 \\
\hline 9 & 1800 & 9 & 0 & 0 & 0 & 0 & 0 & 0 & 0 \\
\hline 10 & 1900 & 10 & 0 & 0 & 0 & 0 & 0 & 0 & 0 \\
\hline 11 & 2000 & 11 & 0 & 0 & 0 & 0 & 0 & 0 & 0 \\
\hline 12 & 2100 & 12 & 0 & 0 & 0 & 0 & 0 & 0 & 0 \\
\hline 13 & 2200 & 14 & 0 & 0 & 0 & 0 & 0 & 0 & 0 \\
\hline 14 & 2300 & 16 & 0 & 0 & 0 & 0 & 0 & 0 & 0 \\
\hline 15 & 2400 & 19 & 0 & 0 & 0 & 0 & 0 & 1 & 3 \\
\hline 16 & 2500 & 22 & 0 & 0 & 0 & 0 & 0 & 0 & 0 \\
\hline 21 & $\# 3000$ & 27 & 1 & 1 & 1 & 1 & 1 & 0 & 0 \\
\hline 37 & $\# 4600$ & 43 & 1 & 1 & 1 & 1 & 1 & 1 & 0 \\
\hline & TLR (\%) & & 4.2 & 1.4 & 1.3 & 0.9 & 0.52 & 0.45 & 0.42 \\
\hline & TF & & 150 & 160 & 160 & 157 & 145 & 138 & 133 \\
\hline
\end{tabular}

Note: the number of rolls $=135$.

TABLE 6: The range and midpoint of $R$.

\begin{tabular}{llccccc}
\hline Range of $R$ & 0 & $-1 \sim-2$ & $-3 \sim-8$ & $-9 \sim-86$ & $-87 \sim-533$ & $-534 \sim-\infty$ \\
\hline Midpoint & 0 & -1 & -5 & -47 & -310 & -1000 \\
\hline
\end{tabular}

TABLE 7: Information of $\mathrm{pq}_{k}$ for simulated examples.

\begin{tabular}{cccccccc}
\hline$k$ & $\mathrm{pw}_{k}$ & $f_{k}$ & 1 & 2 & 3 & 4 & 5 \\
\hline 1 & 1000 & 1 & 46 & 2 & 6 & 37 & 9 \\
2 & 1100 & 2 & 3 & 15 & 2 & 47 & 41 \\
3 & 1200 & 3 & 33 & 11 & 31 & 5 & 33 \\
4 & 1300 & 4 & 22 & 21 & 22 & 42 & 27 \\
5 & 1400 & 5 & 39 & 12 & 20 & 36 & 1 \\
6 & 1500 & 6 & 46 & 25 & 6 & 31 & 2 \\
7 & 1600 & 7 & 22 & 26 & 21 & 38 & 12 \\
8 & 1700 & 8 & 34 & 21 & 34 & 6 & 41 \\
9 & 1800 & 9 & 30 & 25 & 31 & 32 & 29 \\
10 & 1900 & 10 & 38 & 14 & 44 & 26 & 48 \\
11 & 2000 & 11 & 1 & 20 & 12 & 48 & 22 \\
12 & 2100 & 12 & 23 & 42 & 37 & 42 & 16 \\
13 & 2200 & 14 & 12 & 20 & 48 & 11 & 48 \\
14 & 2300 & 16 & 6 & 31 & 33 & 2 & 19 \\
15 & 2400 & 19 & 27 & 12 & 41 & 28 & 30 \\
16 & 2500 & 22 & 14 & 38 & 41 & 13 & 27 \\
\hline
\end{tabular}

According to Table 5, when $R=1$, TLR increased and $\{1000,21\}$ changed to $\{1000,11\}$. This was primarily because
TABLE 8: The results of TF and TLR for CSP, TLMP, and FAP.

\begin{tabular}{|c|c|c|c|c|c|c|}
\hline \multirow{2}{*}{ Method } & \multirow{2}{*}{ Measure } & \multicolumn{5}{|c|}{ Case } \\
\hline & & 1 & 2 & 3 & 4 & 5 \\
\hline \multirow{2}{*}{ CSP } & $\mathrm{TF}$ & 94 & 98 & 10 & 43 & 0 \\
\hline & TLR (\%) & 0 & 1.1 & 1.6 & 0.5 & 1.8 \\
\hline \multirow{2}{*}{ TLMP } & TF & 49 & 19 & 6 & 10 & 9 \\
\hline & TLR (\%) & 0 & 0.15 & 1.2 & 0.13 & 1.2 \\
\hline \multirow{2}{*}{$\begin{array}{l}\text { FAP } \\
(R=0)\end{array}$} & TF & 109 & 115 & 25 & 65 & 26 \\
\hline & TLR (\%) & 0 & 1 & 1.6 & 0.16 & 1.4 \\
\hline \multirow{2}{*}{$\begin{array}{l}\text { FAP } \\
(R=-1000)\end{array}$} & $\mathrm{TF}$ & 109 & 19 & 6 & 20 & 9 \\
\hline & TLR (\%) & 0 & 0.15 & 1.2 & 0.13 & 1.2 \\
\hline Rolls & & 144 & 141 & 183 & 162 & 162 \\
\hline
\end{tabular}

trim loss was equivalent to the flexible coefficient of $\{1000\}$, causing the stock capacity of $\{1000\}$ to decrease. Thus, when $0 \leq R<1$ is defined, we can directly use $R=0$ for solution identification.

When $R<0$, we observed that the TF gradually reduced from 160 to 133 and the TLR reduced from 1.4 to 0.42 . These results suggest that, when $R$ has a value less than 0 , the TF decreases and the TLR declines. Regarding flexible stock, we found that, when $R$ ranged between -1 and -2 , 
TABLE 9: Stock information for CSP, TLMP, and FAP.

\begin{tabular}{|c|c|c|}
\hline Case & Method & Solution \\
\hline \multirow{4}{*}{1} & CSP & $\{1100,1\}\{1500,1\}\{\# 4600,2\}$ \\
\hline & TLMP & $\{1000,3\}\{1100,2\}\{2200,3\}$ \\
\hline & $\operatorname{FAP}(R=0)$ & $\{\# 2600,1\}\{\# 4600,2\}$ \\
\hline & $\operatorname{FAP}(R=-1000)$ & $\{\# 2600,1\}\{\# 4600,2\}$ \\
\hline \multirow{4}{*}{2} & CSP & $\{1000,1\}\{2000,1\}\{\# 4600,2\}$ \\
\hline & TLMP & $\{1000,17\}\{1100,1\}$ \\
\hline & $\operatorname{FAP}(R=0)$ & $\{\# 3200,1\}\{\# 4600,2\}$ \\
\hline & $\operatorname{FAP}(R=-1000)$ & $\{1000,17\}\{1100,1\}$ \\
\hline \multirow{4}{*}{3} & CSP & $\{1300,1\}\{1500,1\}$ \\
\hline & TLMP & $\{1000,6\}$ \\
\hline & $\operatorname{FAP}(R=0)$ & $\{\# 2800,1\}$ \\
\hline & $\operatorname{FAP}(R=-1000)$ & $\{1000,6\}$ \\
\hline \multirow{4}{*}{4} & CSP & $\{\# 4600,1\}$ \\
\hline & TLMP & $\{1000,6\}\{1300,1\}$ \\
\hline & $\operatorname{FAP}(R=0)$ & $\{2500,1\}\{\# 4600,1\}$ \\
\hline & $\operatorname{FAP}(R=-1000)$ & $\{1000,4\}\{1100,1\}\{2200,1\}$ \\
\hline \multirow{4}{*}{5} & CSP & NA \\
\hline & TLMP & $\{1000,3\}\{1500,1\}$ \\
\hline & $\operatorname{FAP}(R=0)$ & $\{\# 2900,1\}$ \\
\hline & $\operatorname{FAP}(R=-1000)$ & $\{1000,3\}\{1500,1\}$ \\
\hline
\end{tabular}

Note: \{paper width, stock quantity\}.

the production capacity of $\{1000\}$ was 22 ; subsequently, as $R$ decreased to between -3 and -8 and -534 and $-\infty$, the production capacity of $\{1000\}$ increased to 28 and to 37 , respectively. These results suggest that, as $R$ decreases, the allocation of stock gradually coagulates at a lower leniency, negating the effects of extended stock. The decrease in TF from 160 to 133 implies that the degree of permitted flexibility for adjusting stock had already diminished. Therefore, we suggest that $R$ be maintained within a range between $-\infty$ and 0 .

Because the trim loss value at each interval of $R$ is a fixed value, we selected the medians of each interval and tabulated them into Table 6, which enabled us to select the desired results. Consequently, the number of medians can be defined by decision makers based on actual conditions.

4.2. Simulated Examples. To verify the superiority of the flexible stock and trim loss produced by using FAP over those produced using CSP and TLMP, we selected 5 Cases for comparison, and randomly obtained the $\mathrm{pq}_{k}$ (where $k=$ $1,2, \ldots, 16)$, which was achieved by using the RANDBETWEEN function in Microsoft Office Excel 2007. The range of this function was set between $0,1,2, \ldots, 50$ (see Table 7). The optimization calculations were then performed for FAP, CSP, and TLMP.

We compared $\operatorname{FAP}(R=0), \operatorname{FAP}(R=-1000), \mathrm{CSP}$, and TLMP, and the results were tabulated in Table 8 . Because using FAP necessitates the consideration of the flexible coefficients, $\operatorname{FAP}(R=0)$ should effectively reduce TLR when an excessively large CSP's TLR value is produced. Cases 2, 4, and 5 verified that FAP reduced CSP's TLR. FAP $(R=-1000)$ and TLMP were then examined to determine whether FAP's TLR presented similarities with TLMP's TLR. Consequently, the TLR values observed in all the 5 Cases were consistent.

Subsequently, we endeavored to determine whether FAP could effectively increase the flexibility of stock adjustment (see Table 9). The $\operatorname{FAP}(R=0)$ for Cases 1 and 3 indicated that the stock leniency demonstrated a merging action. In addition, the extended stock was used in all of the case samples. Furthermore, uncut rolls $\{\# 4600\}$ were presented in Cases $1,2,4$, and 5 . Because $R=0$ is the lowest production capacity model, this model is equivalent to CSP. The $\operatorname{FAP}(R=$ $-1000)$ for Cases 2, 3, and 5 was similar. However, $\operatorname{FAP}(R=$ $-1000)$ presented increased stock adjustment flexibility and extended stock usage in Cases 1 and 4 . Thus, $\operatorname{FAP}(R=0)$ can effectively reduce CSP's TLR and increase stock adjustment flexibility when TLR is at a minimum level. The TLR in $\operatorname{FAP}(R=-1000)$ was equivalent to that of TLMP, which increased stock adjustment flexibility.

A sensitivity analysis was employed to determine the performance of FAP in the 5 Cases and the influence of $R$ on TLR and TF. Consequently, $R$ was set at $1,0,-1, \ldots,-\infty$, where $R$ was an integer. The results are tabulated in Table 10.

The medians tabulated in Table 6 were used for data reconstruction and the results are presented in Table 11. Subsequently, we collected the $R$ values at each interval for Cases 1, 3, 4, and 5. For Case 2, we were unable to collect the $R$ values at intervals of $-55--79,-80--124$, and $-125-$ -156 . Decision makers can determine whether they wish to incorporate the medians at these intervals or not; however, this method of incorporating medians can be used to control the majority of TLR changes.

\section{Conclusion}

The results of the case study analysis indicate that $\operatorname{FAP}(R=0)$ was similar to CSP in that both methods could be used to determine the minimal production capacity and the maximal, flexible adjusted stock. Because of the unique production characteristics of industrial-use paper, using the CSP method may produce full rolls and, thus, cannot obtain optimized trim loss problems. Similar to the CSP method, $\operatorname{FAP}(R=$ -1000) generates stock that cannot be flexibly adjusted, despite possessing minimal trim loss. Furthermore, CSP and TLMP failed to control the changes of TLR; therefore, FAP can utilize $R$ to control and maintain TLR in a range between CSP and TLMP'TLR. This approach eliminates the trim loss problem exhibited in CSP and the adjustability problem exhibited in TLMP and allows decision makers to effectively control stock and trim loss according to actual situations.

Future research may consider solving extending stock in stock allocation. In addition, the cost effects during the production process should be addressed.

\section{Notations}

$i$ : $\quad$ The index number $(i=1,2, \ldots, m)$ and $m$

is the number of stock/order widths

$\mathrm{sw}_{i}$ : A stock width with $i=1,2, \ldots, m$

ow $_{i}$ : An order width with $i=1,2, \ldots, m$ 
TABLE 10: The sensitivity analysis of coefficient $R$ for all cases using FAP.

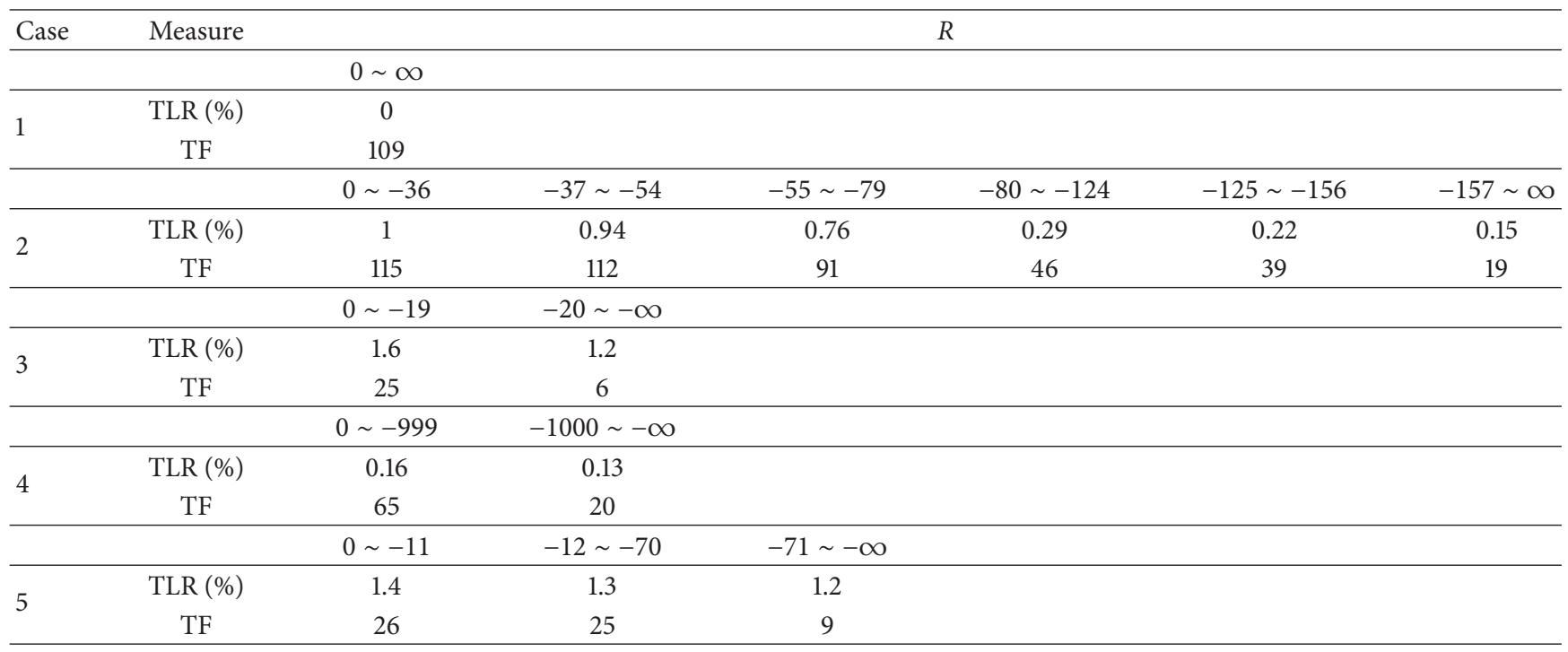

TABLE 11: The results of fixed values of $R$ for all cases using FAP.

\begin{tabular}{lccccccc}
\hline \multirow{2}{*}{ Case } & Measure & \multicolumn{7}{c}{$R$} \\
& & 0 & -1 & -5 & -47 & -310 & -1000 \\
\hline \multirow{2}{*}{1} & TLR (\%) & 0 & 0 & 0 & 0 & 0 & 0 \\
& TF & 109 & 109 & 109 & 109 & 109 & 109 \\
\hline \multirow{2}{*}{2} & TLR (\%) & 1 & 1 & 1 & 0.94 & 0.15 & 0.15 \\
& TF & 115 & 115 & 115 & 112 & 19 & 19 \\
\hline \multirow{2}{*}{3} & TLR (\%) & 1.6 & 1.6 & 1.6 & 1.2 & 1.2 & 1.2 \\
& TF & 25 & 25 & 25 & 6 & 6 & 6 \\
\hline \multirow{2}{*}{4} & TLR (\%) & 0.16 & 0.16 & 0.16 & 0.16 & 0.16 & 0.13 \\
& TF & 65 & 65 & 65 & 65 & 65 & 20 \\
\hline \multirow{2}{*}{5} & TLR (\%) & 1.4 & 1.4 & 1.4 & 1.3 & 1.2 & 1.2 \\
& TF & 26 & 26 & 26 & 25 & 9 & 9 \\
\hline
\end{tabular}

$d_{i}: \quad$ Demand for ow with $_{i}=1,2, \ldots, m$

L: $\quad$ Reel width

NR: The number of rolls for a reel

GAP: The difference between two paper widths

$\mathrm{ew}_{e}$ : An extending production width, where $e$ is the index number

$(e=m+1, m+2, \ldots, e x)$ and

ex $=m+\left(L-\mathrm{sw}_{m}\right) / \mathrm{GAP}$

$\mathrm{pw}_{k}$ : A production width, where $k$ is the index number $(k=1,2, \ldots$, ex $)$

$\mathrm{pq}_{k}$ : Quantity for the production width, $k=1,2, \ldots$, ex

$f_{k}$ : Flexible coefficient for the production width $\mathrm{pw}_{k}$, with $k=1,2, \ldots$, ex

$R: \quad$ Flexible coefficient for trim loss

SRQ: Production scheduling of reel quantity

UB: Upper bound for trim loss $a_{k r}:$ The number of widths $k$ in pattern $r$

$x_{r}$ : The number of patterns $r$, where $r$ is the index number $(r=1,2, \ldots, t)$ and $t$ is the number of patterns.

\section{Conflict of Interests}

The authors declare that they have no conflict of interests.

\section{References}

[1] H. Stadtler, "A one-dimensional cutting stock problem in the aluminium industry and its solution," European Journal of Operational Research, vol. 44, no. 2, pp. 209-223, 1990.

[2] M. Gradǐsar, J. Jesenko, and G. Resinovič, "Optimization of roll cutting in clothing industry," Computers and Operations Research, vol. 24, no. 10, pp. 945-953, 1997.

[3] L. O. Morgan, A. R. Morton, and R. L. Daniels, "Simultaneously determining the mix of space launch vehicles and the assignment of satellites to rockets," European Journal of Operational Research, vol. 172, no. 3, pp. 747-760, 2006.

[4] G. Wäscher, H. Haußner, and H. Schumann, "An improved typology of cutting and packing problems," European Journal of Operational Research, vol. 183, no. 3, pp. 1109-1130, 2007.

[5] A. Dikili, A. Takinaci, and N. Pek, "A new heuristic approach to one-dimensional stock-cutting problems with multiple stock lengths in ship production," Ocean Engineering, vol. 35, no. 7, pp. 637-645, 2008.

[6] A. Abuabara and R. Morabito, "Cutting optimization of structural tubes to build agricultural light aircrafts," Annals of Operations Research, vol. 169, no. 1, pp. 149-165, 2009.

[7] M. Gradišar, G. Resinovič, and M. Kljajić, "Evaluation of algorithms for one-dimensional cutting," Computers \& Operations Research, vol. 29, no. 9, pp. 1207-1220, 2002.

[8] P. C. Gilmore and R. E. Gomory, "A linear programming approach to the cutting-stock problem," Operations Research, vol. 9, no. 6, pp. 849-859, 1961. 
[9] P. C. Gilmore and R. E. Gomory, "A linear programming approach to the cutting stock problem, part II," Operations Research, vol. 11, no. 3, pp. 863-888, 1963.

[10] W. Gochet and M. Vandebroek, "A dynamic programming based heuristic for industrial buying of cardboard," European Journal of Operational Research, vol. 38, no. 1, pp. 104-112, 1989.

[11] C. H. Dağli, "Knowledge-based systems for cutting stock problems," European Journal of Operational Research, vol. 44, no. 2, pp. 160-166, 1990.

[12] H. Foerster and G. Wäscher, "Simulated annealing for order spread minimization in sequencing cutting patterns," European Journal of Operational Research, vol. 110, no. 2, pp. 272-281, 1998.

[13] P. H. Vance, "Branch-and-price algorithms for the onedimensional cutting stock problem," Computational Optimization and Applications, vol. 9, no. 3, pp. 211-228, 1998.

[14] M. Gradišar, M. Kljajić, G. Resinovič, and J. Jesenko, "A sequential heuristic procedure for one-dimensional cutting," European Journal of Operational Research, vol. 114, no. 3, pp. 557-568, 1999.

[15] M. Sakawa, I. Nishizaki, and M. Hitaka, "Interactive fuzzy programming for multi-level 0-1 programming problems through genetic algorithms," European Journal of Operational Research, vol. 114, no. 3, pp. 580-588, 1999.

[16] S. M. A. Suliman, "Pattern generating procedure for the cutting stock problem," International Journal of Production Economics, vol. 74, no. 1-3, pp. 293-301, 2001.

[17] H. B. Amor, J. Desrosiers, and J. M. Valério de Carvalho, "Dual-optimal inequalities for stabilized column generation," Operations Research, vol. 54, no. 3, pp. 454-463, 2006.

[18] P. Trkman and M. Gradisar, "One-dimensional cutting stock optimization in consecutive time periods," European Journal of Operational Research, vol. 179, no. 2, pp. 291-301, 2007.

[19] W.-C. Weng and T.-C. Sung, "Optimization of a line-cutting procedure for ship hull construction by an effective tabu search," International Journal of Production Research, vol. 46, no. 21, pp. 5935-5949, 2008.

[20] C. Alves and J. M. Valério de Carvalho, "Accelerating column generation for variable sized bin-packing problems," European Journal of Operational Research, vol. 183, no. 3, pp. 1333-1352, 2007.

[21] S. L. Nonås and A. Thorstenson, "Solving a combined cuttingstock and lot-sizing problem with a column generating procedure," Computers and Operations Research, vol. 35, no. 10, pp. 3371-3392, 2008.

[22] H. Reinersten and T. W. M. Vossen, "The one-dimensional cutting stock problem with due dates," European Journal of Operational Research, vol. 201, no. 3, pp. 701-711, 2010.

[23] K. Matsumoto, S. Umetani, and H. Nagamochi, "On the onedimensional stock cutting problem in the paper tube industry," Journal of Scheduling, vol. 14, no. 3, pp. 281-290, 2011.

[24] J. Erjavec, M. Gradisar, and P. Trkman, "Assessment of stock size to minimize cutting stock production costs," International Journal of Production Economics, vol. 135, no. 1, pp. 170-176, 2012.

[25] M. H. M. A. Jahromi, R. Tavakkoli-Moghaddam, A. Makui, and A. Shamsi, "Solving an one-dimensional cutting stock problem by simulated annealing and tabu search," Journal of Industrial Engineering International, vol. 8, no. 1, pp. 24-31, 2012.

[26] A. Mobasher and A. Ekici, "Solution approaches for the cutting stock problem with setup cost," Computers \& Operations Research, vol. 40, no. 1, pp. 225-235, 2013.
[27] G. C. Wang, C. P. Li, J. Lv, X. X. Zhao, and H. Y. Cuo, "An efficient algorithm design for the one-dimensional cuttingstock problem," Advanced Materials Research, vol. 602-604, pp. 1753-1756, 2013.

[28] H. H. Yanasse, "A review of three decades of research on some combinatorial optimization problems," Pesquisa Operacional, vol. 33, no. 1, pp. 11-36, 2013.

[29] L. Kos and J. Duhovnik, "Cutting optimization with variablesized stock and inventory status data," International Journal of Production Research, vol. 40, no. 10, pp. 2289-2301, 2002.

[30] S. Dimitriadis and E. Kehris, "Cutting stock optimization in custom door and window manufacturing industry," International Journal of Decision Sciences, Risk and Management, vol. 1, no. 1, pp. 66-80, 2009.

[31] A. C. Cherri, M. N. Arenales, and H. H. Yanasse, "The onedimensional cutting stock problem with usable leftover-a heuristic approach," European Journal of Operational Research, vol. 196, no. 3, pp. 897-908, 2009.

[32] K. C. Poldi and M. N. Arenales, "Heuristics for the onedimensional cutting stock problem with limited multiple stock lengths," Computers and Operations Research, vol. 36, no. 6, pp. 2074-2081, 2009.

[33] Y. Cui and Y. Yang, "A heuristic for the one-dimensional cutting stock problem with usable leftover," European Journal of Operational Research, vol. 204, no. 2, pp. 245-250, 2010.

[34] S. A. Araujo, A. A. Constantino, and K. C. Poldi, "An evolutionary algorithm for the one-dimensional cutting stock problem," International Transactions in Operational Research, vol. 18, no. 1, pp. 115-127, 2011.

[35] A. C. Cherri, M. N. Arenales, and H. H. Yanasse, "The usable leftover one-dimensional cutting stock problem-a priorityin-use heuristic," International Transactions in Operational Research, vol. 20, no. 2, pp. 189-199, 2013.

[36] F. K. Wang and F. T. Liu, "A new decision model for reducing trim loss and inventory in the paper industry," Journal of Applied Mathematics, vol. 2014, Article ID 987054, 10 pages, 2014.

[37] Lingo Software, Version 11, Lindo Systems, Inc, Chicago, Ill, USA, 2009. 


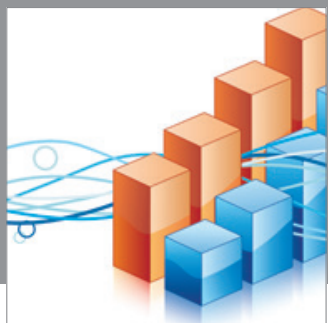

Advances in

Operations Research

mansans

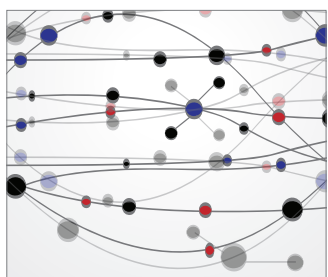

The Scientific World Journal
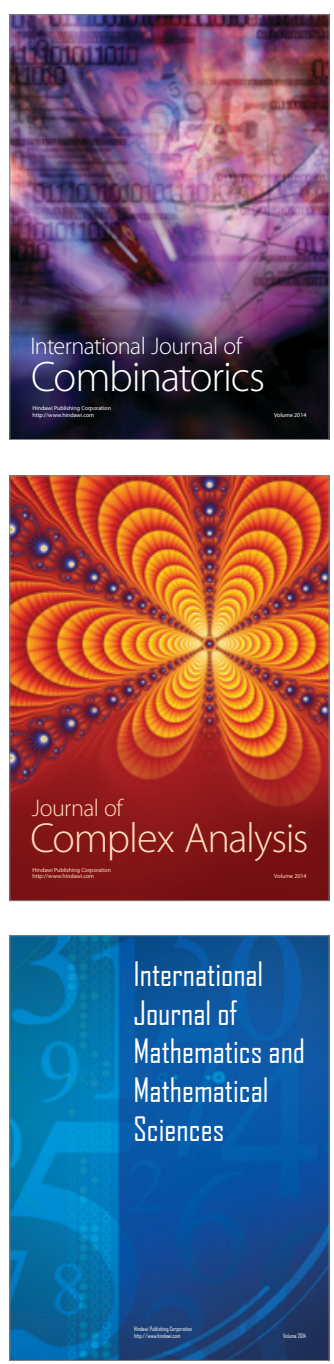
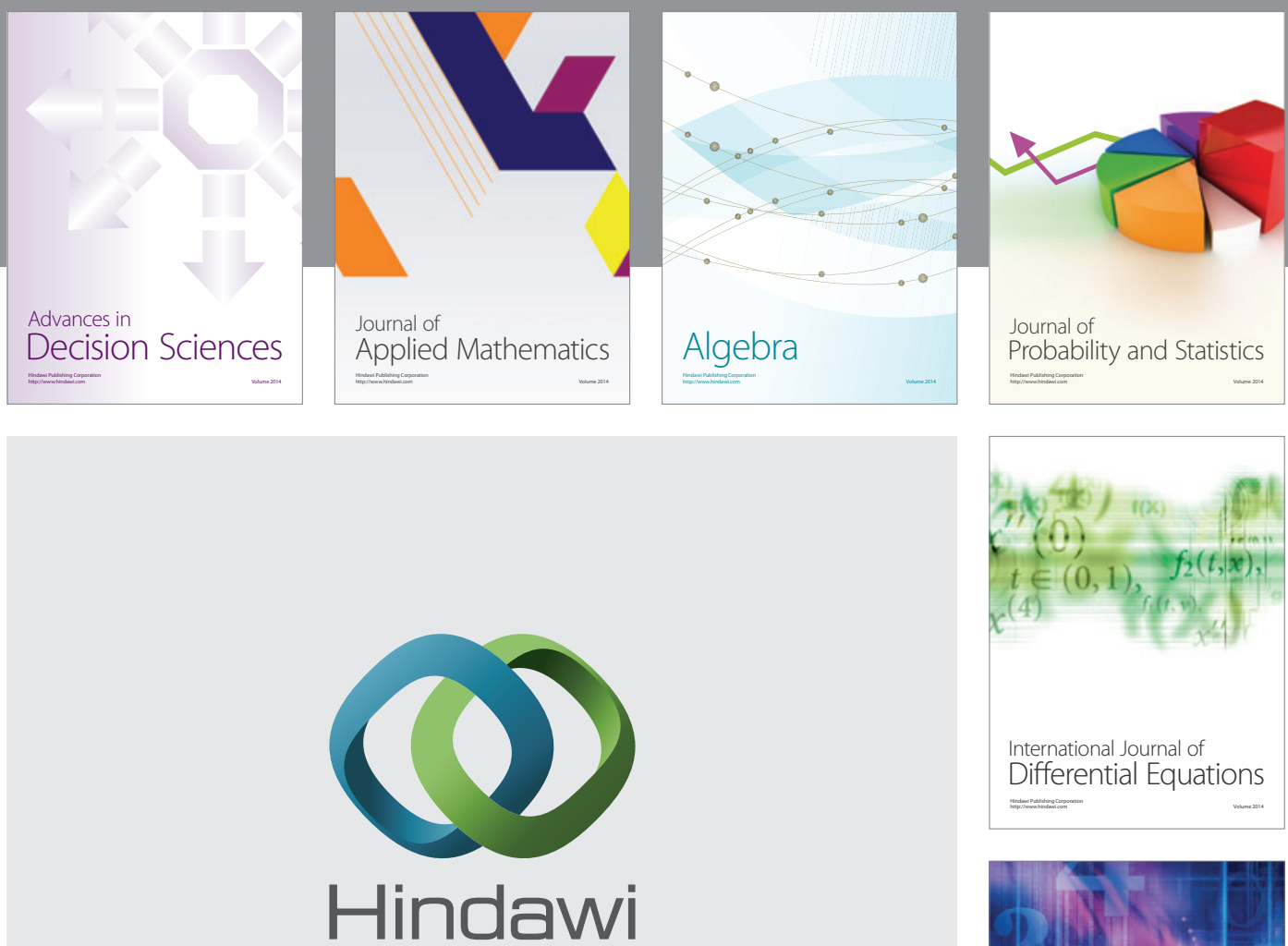

Submit your manuscripts at http://www.hindawi.com
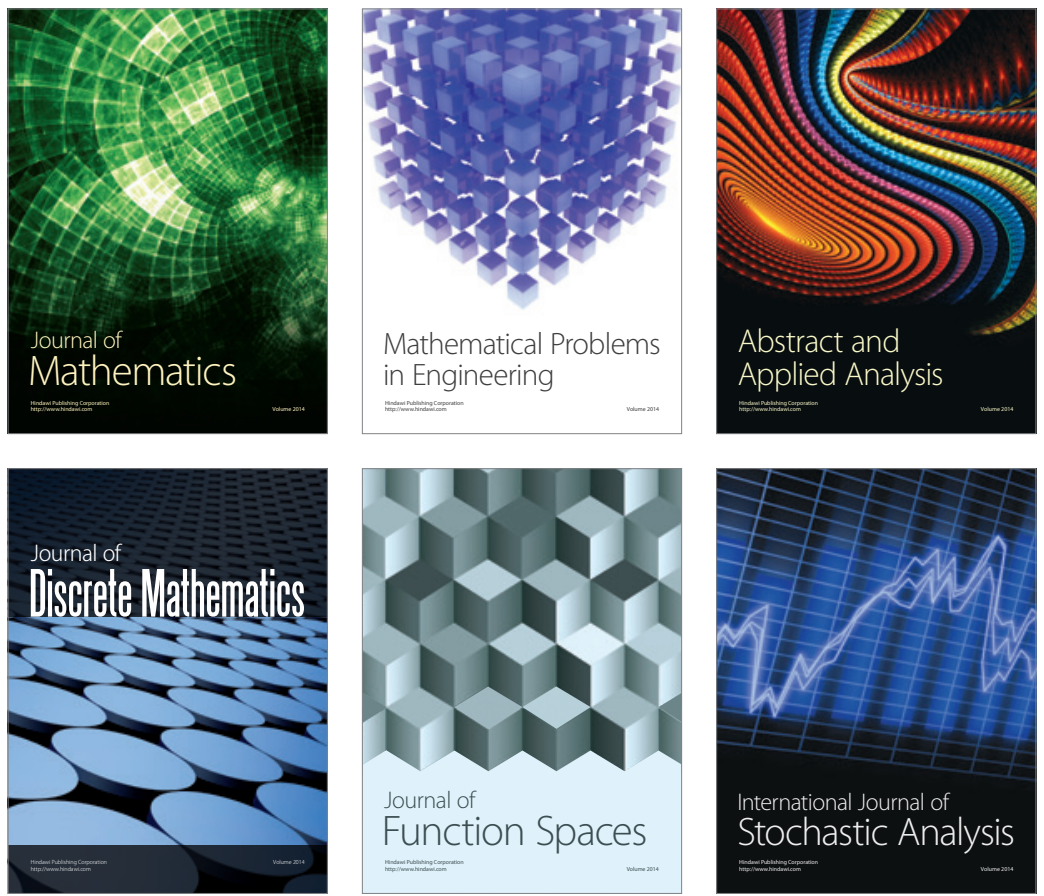

Journal of

Function Spaces

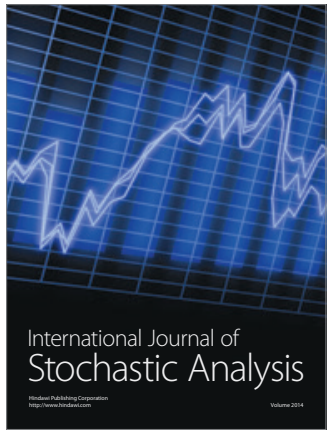

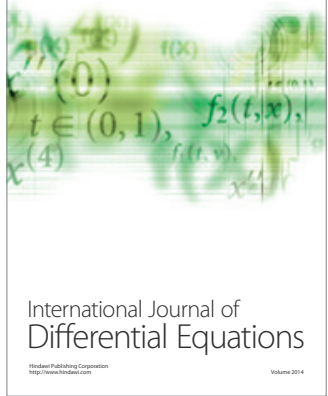
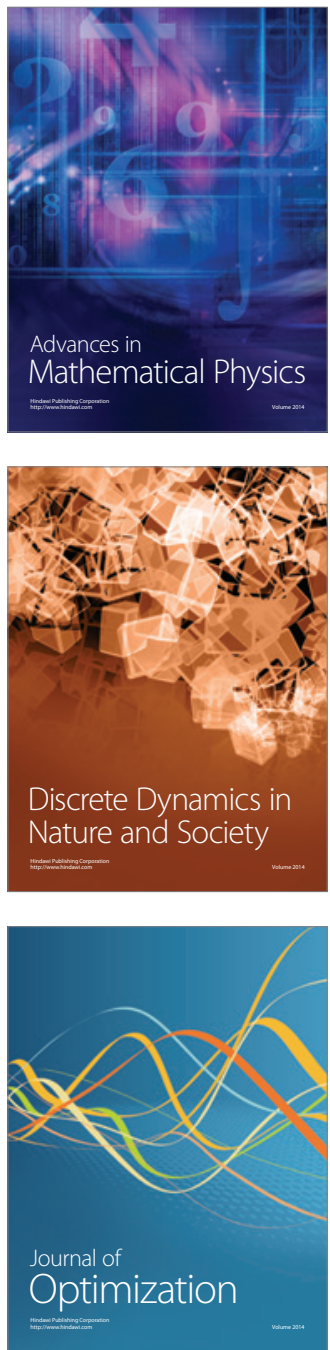